\title{
Integrated disease management improves one-year quality of life in primary care COPD patients: a controlled clinical trial
}

\author{
*Niels H Chavannes ${ }^{\mathrm{a}, \mathrm{b}}$, Marlous Grijsen ${ }^{\mathrm{b}}$, Marjan van den Akker ${ }^{\mathrm{b}}$, Huub Schepersc, \\ Maddy Nijdam, Brian Tiep ${ }^{d}$, Jean Muris ${ }^{b}$ \\ a Public Health and Primary Care, Leiden University Medical Center, Leiden, The Netherlands \\ ${ }^{b}$ Department of General Practice, Maastricht University, Maastricht, The Netherlands \\ ' Well-being Health Center Bocholtz-Simpeld, Bocholtz, The Netherlands \\ ${ }^{\mathrm{d}}$ Respiratory Institute, Irwindale, California, USA
}

Received 30th June 2008; resubmitted 19th September 2008; accepted 19th October 2008; online 14th January 2009

\begin{abstract}
Aim: To assess the long-term effectiveness of an integrated disease management (IDM) program (consisting of optimal medication, reactivation, education, and exacerbation management) in primary care patients with chronic obstructive pulmonary disease (COPD).

Method: Controlled trial comparing the effects of IDM on quality of life - assessed by the St. George's Respiratory Questionnaire (SGRQ) - in primary care COPD patients. The minimal clinically important change on the SGRQ was accepted as being -4 points. Baseline and oneyear differences were compared using paired sample T-tests. The differential effects of an $\mathrm{FEV}_{1} / \mathrm{FVC}$ ratio $<0.7$ and dyspnoea as assessed by the Medical Research Council (MRC) Dyspnoea scale were investigated.

Results: The average age of subjects was 63 years, with an average post-bronchodilator $\mathrm{FEV}_{1}$ of $67 \%$ predicted, average $\mathrm{FEV} \mathrm{V}_{1} / \mathrm{FVC}$ ratio of 0.65 , a mean of 35 pack-years smoking, and $63 \%$ were male. No significant differences existed between groups at baseline. After one year of IDM, SGRQ had improved by -4.6 points ( $95 \% \mathrm{Cl},-7.2$ to $-2.0 ; \mathrm{p}=0.001)$ in the intervention group, versus -0.7 points $(95 \% \mathrm{Cl}$, 3.0 to $1.6 ; p=0.6)$ in the usual care group. In patients with an $\mathrm{FEV}_{1} / \mathrm{FVC}$ ratio $<0.7$, SGRQ improved by -5.9 points $(95 \% \mathrm{Cl},-9.6$ to -2.2 ; $\mathrm{p}=0.002)$ in the IDM group, while in the usual care group SGRQ improved by -0.8 points $(95 \% \mathrm{Cl},-4.1$ to $2.4 ; p=0.6)$. In patients with an MRC Dyspnoea score $>2$ and $F E V_{1} / F V C<0.7$, SGRQ improved by -13.4 points $(95 \% \mathrm{Cl},-20.8$ to $-6.1 ; p=0.002)$ in the IDM group, versus -0.3 points $(95 \% \mathrm{Cl},-5.5$ to $4.9 ; \mathrm{p}=0.9)$ in the usual care group.

Conclusion: In this study, IDM improved one-year quality of life in primary care COPD patients, compared to usual care. The improvement in SGRQ was both clinically relevant and statistically significant, and was greatest in patients with FEV $1 / F V C<0.7$ and MRC Dyspnoea score $>2$.

(C) 2009 General Practice Airways Group. All rights reserved.

NH Chavannes, et al. Prim Care Resp J 2009; 18(3): 171-176.

doi:10.3132/pcrj.2009.00003
\end{abstract}

Keywords COPD, quality of life, questionnaire, SGRQ, FEV $1 / F V C$ ratio, MRC Dyspnoea score

\section{Background}

Since the concept of COPD as a treatable disease was introduced in the 2004 ATS/ERS COPD position paper, ${ }^{1}$ several studies have shown beneficial effects of medication on exercise tolerance, ${ }^{2}$ quality of life, ${ }^{3}$ and exacerbations. ${ }^{4}$ The size of the effect, however, was usually modest, and the study populations often consisted of selected moderate to very severe patients, considerably reducing the external validity for primary care patients. ${ }^{5}$

The importance of non-medical interventions such as smoking cessation and physical activity enhancement was underscored recently, when 20-year follow-up data were published $^{6,7}$ showing that daily exercise reduces lung function decline (and consequently the risk of developing COPD),

\footnotetext{
* Corresponding author: Dr Niels H Chavannes, Public Health and Primary Care, Leiden University Medical Center, Hippocratespad 21, PO Box 9600, Zone V0-P, Leiden, 2300 RC, The Netherlands. Tel: +31 715268444 Fax: +31 715268259 E-mail: n.h.chavannes@lumc.nl
} 
hospitalisation and even respiratory mortality by 30 to $40 \%$ a protective effect which until now remained reserved for smoking cessation alone, ${ }^{8}$ since medical interventions have failed to show significant effects on mortality. ${ }^{4}$ Selfmanagement programmes can also reduce hospitalisations by $40 \%$, but this has until now only been demonstrated in patients with severe COPD. ${ }^{9}$

In daily practice, the majority of patients are treated in primary care, where most suffer from mild to moderate COPD. ${ }^{10}$ Therefore, pragmatic primary care studies in which optimal medical and non-medical treatments are combined into an integrated disease management (IDM) intervention are needed, but to our knowledge have been lacking to this date.

From longstanding clinical observations during the development of disease management programmes in primary care COPD patients, we deduced a hypothesis: the potential for improvement in quality of life (QoL) seems greatest when lung function is relatively intact, while symptoms of dyspnoea are already considerable. We have therefore performed a controlled clinical trial in primary care COPD patients to assess the effects of an IDM programme on long term diseasespecific QoL.

\section{Method}

Two primary health care centres serving two separate villages in the southern part of the Netherlands were recruited for epidemiological reasons: both had very similar patient populations with comparable regional living conditions, but these were traditionally self-sufficient communities with little risk of intervention contamination.

Due to pre-existing national primary care guidelines for the diagnosis and treatment of COPD, health care providers in the two centres were working in a comparable fashion before commencing the study; this changed, however, when the IDM model was introduced in the intervention setting during which time usual care was maintained in the control health centre. The control health centre, lying on the other side of a hill separating the two villages, was kept strictly unaware of the new treatment strategies, on the condition that swift and facilitated implementation would occur after the study was completed successfully. The IDM programme was based largely on recommendations from the ATS/ERS COPD Standards, ${ }^{10}$ with additions from earlier work on disease management, ${ }^{11}$ and included optimal medication prescribing and adherence monitoring, rapid action plans for exacerbations, personalised physical activity training programmes (at least three sessions of at least 40 minutes of physical activity per week over three months) and continuous self-management education including personal goal-setting by motivational interviewing techniques.
An integrated COPD management team was created, comprising two specialised physiotherapists, a respiratory nurse, a physician assistant, a dietician, a pharmacist, a supervising primary care physician, and a logistical manager, who took charge of the monthly team meetings. A standardised treatment protocol was written by all team members, each taking responsibility for their respective areas of expertise, which could be optimally tailored to individual patient needs.

Patients were recruited on the basis of an existing diagnosis of COPD, with chronic respiratory complaints in the absence of a prior history of asthma or atopy, and had to fulfil former national guideline lung function criteria with a postbronchodilator $\mathrm{FEV}_{1}<80 \%$ predicted and/or a postbronchodilator $\mathrm{FEV}_{1} / \mathrm{FVC}$ ratio $<0.7$. Since the latter requirement was not a diagnostic criterion in the earlier national guideline, it was decided beforehand to investigate the differential effects of both the $\mathrm{FEV}_{1} / \mathrm{FVC}$ ratio $<0.7$ and the patients' Medical Research Council (MRC) Dyspnoea scores. Exclusion criteria were limited, and consisted of rapidly progressing or terminal disease, immobility, substance abuse, or inability to fill in questionnaires.

- Power calculation (power of $80 \%$ with $\alpha=0.05$ ) indicated that $2 \times 75$ patients were needed to detect a clinically relevant change in QoL on the St George's Respiratory Questionnaire (SGRQ), ${ }^{12}$ including 20\% lost to follow-up. We used the Dutch self-administered version of the SGRQ, and considered a -4 unit change as the minimum clinically important difference (MCID) for within-group comparison. ${ }^{13}$ As a second disease-specific measure of QoL we used the Dutch diary version of the Clinical COPD Questionnaire (CCQ), ${ }^{14}$ which is especially useful in the primary care setting and which shows an MCID of -0.4 points. ${ }^{15}$ The MRC Dyspnoea score was used to assess dyspnoea, using the original 5-point scale. ${ }^{16}$ By simply naming daily physical activities like walking or cycling, we obtained an impression of inactive lifestyle at baseline. Current and past smoking was assessed by asking systematically for smoking history and calculating pack-years. We did not use biochemical methods to validate smoking status as the primary outcome was not smoking cessation.

Data were analysed with SPSS version 13, using independent T-tests and chi-square tests for baseline characteristics comparison. We only used paired sample Ttests for prospective within-group comparisons, since this pragmatic controlled clinical trial was not randomised and thus head-to-head comparison was not deemed statistically sound. The regional Medical Ethics Committee of the Atrium Medical Centre Heerlen approved of the study protocol, while all participating patients gave their written informed consent. 
Table 1. Baseline characteristics of intervention versus control group*

\begin{tabular}{|c|c|c|c|}
\hline & Intervention $(n=79)$ & Control $(n=73)$ & $\mathrm{p}$-value\# \\
\hline Age (yrs) & $64(11)$ & $63(11)$ & 0.53 \\
\hline Gender (\% male) & 59 & 67 & 0.32 \\
\hline Pack-years (n) & $37(24)$ & $33(17)$ & 0.33 \\
\hline Current smoking (\%) & 38 & 45 & 0.42 \\
\hline Body Mass Index & $27(5)$ & $27(5)$ & 0.98 \\
\hline Inactive lifestyle (\%) & 45 & 47 & 0.87 \\
\hline FEV 1 pre-BD (\%) & $62(19)$ & $66(16)$ & 0.15 \\
\hline FEV 1 post-BD (\%) & $66(20)$ & $68(17)$ & 0.59 \\
\hline $\mathrm{FEV}_{1} / \mathrm{FVC}$ post-BD & $0.65(.14)$ & $0.65(.13)$ & 0.83 \\
\hline SGRQ -Total & $29.6(20)$ & $34.5(19)$ & 0.12 \\
\hline CCQ & $1.3(.9)$ & $1.6(1)$ & 0.10 \\
\hline$M R C>2(\%)$ & 36 & 32 & 0.61 \\
\hline
\end{tabular}

\section{Results}

We recruited 162 primary care COPD patients, of whom 152 had analysable data - 79 in the intervention group and 73 in the control group. Table 1 shows the study baseline characteristics. There were no significant differences in demographic variables - including smoking behaviour, physical activity, QoL measures, or respiratory symptoms between the intervention and control group. The population consisted of middle-aged patients with largely mild to moderate COPD; 10\% (11/106) had GOLD stage I disease, $61 \%(65 / 106)$ stage II, 25\% (27/106) stage III, and 3\% (3/106) had stage IV disease (data not shown). Subjects had an average smoking history of about 35 pack-years. The proportion of patients with dyspnoea on little exertion or worse, expressed by an MRC score $>2$, was about a third of the study population, while the proportion of patients with an FEV $_{1} /$ FVC ratio $<0.7$ was $70 \%(106 / 152)$.

During the first year of the study, 24 patients (15\%) were lost to follow-up: 11 patients in the intervention group, and 13 in the control group. Most withdrawals (80\%) were due to an unwillingness to fill in questionnaires repeatedly or to attend annually for lung function measurements. No COPDattributable deaths were recorded in either group (data not shown).

Table 2 shows the changes in MRC Dyspnoea scores. After one year, the proportion of patients in the intervention group with MRC >2 had decreased from $36 \%$ to $13 \%$ (relative change $-64 \%)$, whereas the number increased in the control group from $32 \%$ to $44 \%$ (relative increase $+38 \%$ ).

Table 3 shows the one-year changes in SGRQ and CCQ scores in the two groups. There were statistically significant large to moderate improvements of -4.61 and -0.28 , respectively, in the intervention group, while the control group showed non-significant changes of -0.67 and +0.06 , respectively. In patients with an $\mathrm{FEV}_{1} / \mathrm{FVC}$ ratio $<0.7$, (Table 4 ), the effect on SGRQ and CCQ in the intervention group was a large and statistically significant improvement of -5.9 and -0.39 , respectively, while the control group maintained nonsignificant changes of -0.83 and +0.01 , respectively. In patients with both $\mathrm{FEV}_{1} / \mathrm{FVC}<0.7$ and MRC scores $>2$, (Table 5), the effect on SGRQ and CCQ in the intervention group was a very substantial, statistically significant and clinically relevant

Table 2. MRC Dyspnoea Scale; relative changes for the intervention versus control group at 1 year

\begin{tabular}{lllllll} 
& \multicolumn{2}{c}{$\begin{array}{c}\text { Intervention group } \\
\text { baseline / after 1 year }\end{array}$} & Relative change & \multicolumn{2}{c}{$\begin{array}{c}\text { Control group } \\
\text { baseline / after 1 year }\end{array}$} \\
\hline MRC 1-2 & $64 \%$ & $87 \%$ & $+36 \%$ & $68 \%$ & $56 \%$ & $-18 \%$ \\
\hline MRC 3-5 & $36 \%$ & $13 \%$ & $-64 \%$ & $32 \%$ & $44 \%$ & $+38 \%$ \\
\hline
\end{tabular}


NH Chavannes et al.

Table 3. Effect of integrated disease management on quality of life at 1 year*

\begin{tabular}{|c|c|c|c|c|c|c|}
\hline \multirow[b]{2}{*}{ SGRQ } & \multicolumn{2}{|c|}{$\begin{array}{l}\text { Intervention group } \\
1 \text { yr difference / } 95 \% \mathrm{Cl}\end{array}$} & \multirow{2}{*}{$\frac{p \text {-value }}{0.001}$} & \multicolumn{2}{|c|}{$\begin{array}{c}\text { Control group } \\
1 \text { yr difference / 95\% Cl }\end{array}$} & \multirow{2}{*}{$\frac{p \text {-value }}{0.56}$} \\
\hline & -4.61 & {$[-7.2,-2.0]$} & & -0.67 & {$[-3.0,1.6]$} & \\
\hline CCQ & -0.28 & {$[-.44,-.12]$} & 0.001 & +0.06 & {$[-.07,0.2]$} & 0.36 \\
\hline
\end{tabular}

Table 4. Effect of integrated disease management on quality of life in patients with FEV $/$ FVC $<0.7$ at 1 year*

\begin{tabular}{|c|c|c|c|c|c|c|}
\hline \multirow[b]{2}{*}{ SGRQ } & \multicolumn{2}{|c|}{$\begin{array}{c}\text { Intervention group } \\
1 \text { yr difference / } 95 \% \mathrm{Cl}\end{array}$} & \multirow{2}{*}{$\frac{p \text {-value }}{0.002}$} & \multicolumn{2}{|c|}{$\begin{array}{c}\text { Control group } \\
1 \text { yr difference / } 95 \% \mathrm{Cl}\end{array}$} & \multirow{2}{*}{$\frac{p \text {-value }}{0.61}$} \\
\hline & -5.91 & {$[-9.6,-2.2]$} & & -0.83 & {$[-4.1,2.4]$} & \\
\hline CCQ & -0.39 & {$[-.62,-.17]$} & 0.001 & +0.01 & {$[-.17,0.2]$} & 0.88 \\
\hline
\end{tabular}

Table 5. Effect of integrated disease management on quality of life in patients with FEV $1 / F V C<0.7$ and MRC $>2$ at 1 year*

\begin{tabular}{|c|c|c|c|c|c|}
\hline \multirow[b]{2}{*}{ SGRQ } & \multicolumn{2}{|c|}{$\begin{array}{l}\text { Intervention group } \\
1 \mathrm{yr} \text { difference / } 95 \% \mathrm{Cl}\end{array}$} & $p$-value & $\begin{array}{l}\text { sup } \\
95 \% \mathrm{Cl}\end{array}$ & p-value \\
\hline & -13.42 & {$[-20.8,-6.1]$} & 0.002 & {$[-5.5,4.9]$} & 0.91 \\
\hline CCQ & -0.92 & {$[-1.4,-.41]$} & 0.002 & {$[-.29,0.29]$} & 1.0 \\
\hline
\end{tabular}

improvement of -13.42 and -0.92 , respectively, while the control group patients remained showed hon-significant changes of -0.29 and +0.01 respectively.

\section{Discussion}

This pragmatic controlled clinical trial shows that, contrary to common belief, primary care COPD patients can be successfully treated, provided a dedicated multidisciplinary team is in place.

As hypothesised beforehand, the greatest room for improvement seems to be present in patients with mild to moderate disease with an $\mathrm{FEV}_{1} / \mathrm{FVC}<0.7$ but with considerable dyspnoea (MRC score $>2$ ). An important notion seems to be that lung function is still relatively well maintained at that stage, the situation is far from hopeless, and thus physical condition training is of benefit. Possibly, the actual room for improvement is much larger than in patients with (very) severe disease. The physically perceptible change in dyspnoea which occurs within 4-6 weeks of training could be crucial for COPD patients' motivation, as they start to feel that finally there is something that can be done for their disease.

Furthermore, our intervention served different areas of disease expression: personal goals for each individual patient were explicitly formulated and registered on a time-contingent and adjustable basis; exacerbations were tackled at an early stage, since patients were encouraged to seek help within three days of increasing symptoms; group training sessions often led to increased social contacts, as participants were encouraged to start up sporting groups with their peers; and through tailormade education, patients learned about their disease in-depth and about effective therapies and self-management possibilities, which they were often not aware of during years of slowly progressing disease.

All these factors must have contributed to a surprising sense of regaining control, which was reflected in the clinically relevant and statistically significant improvements in QoL measurements. Interestingly, the SGRQ showed the most dramatic improvements across all group comparisons, while the CCQ seemed somewhat less clear. This could possibly be a result of measurements taking place after a year; the most tangible improvements were likely to occur 6-12 weeks after inclusion, and this possibly reflects the sensitive but slightly more volatile character of the CCQ as compared to the SGRQ.

The study setting in two comparable but separate villages that traditionally hardly interact has shown to be effective, since there were no significant baseline differences and very few 
instances of possible contamination were reported. The study team actively looked for intervention contamination during the whole study period, and especially monitored prescription and referral behaviour from the control health centre. Before the study, the control GPs were promised swift and facilitated implementation once the study had ended - if the results turned out to be favourable - but only if the study was completely finished and no attempt was made to interfere with the intervention. It is due to the collective determination of the study team members and a prevailing attitude where these promises were kept, that meant that the contrast between the intervention and control groups was maintained throughout the study.

A favourable factor (for this study) was the low availability and accessibility of rehabilitation facilities in the surrounding areas of the two villages; a bus connection with several stopovers, or a costly taxi journey are required to reach the nearest rehabilitation centre. This certainly meant that our population was in need of our community-based intervention. Nevertheless, this lamentable situation is not unique in the Netherlands (and other countries as well), where insufficient availability and accessibility of nearby rehabilitation facilities is a common and increasing problem at the same time as COPD prevalence is on the rise worldwide.

This study was not designed to optimise smoking cessation, although all smoking participants in both settings were offered guidance according to national COPD guidelines. A nonsignificant cessation difference of $4 \%$ versus $1 \%$ at one year (data not shown) was observed in favour of the intervention group, although this could not explain the significant and clinically relevant change in QoL outcomes. It suggests however, that integrated disease management offers a favourable environment for smoking cessation.

The GOLD definition of obstruction as a fixed ratio of $\mathrm{FEV}_{1} / \mathrm{FVC}$ below 0.7 has only recently been recommended in the revised Dutch national COPD guidelines of 2007. At the time of study commencement, the guideline still recommended an $\mathrm{FEV}_{1}$ of below $80 \%$ predicted for labelling COPD. In our study we applied both criteria, since we aimed to be inclusive rather than exclusive, in order to increase external validity. ${ }^{5}$ Interestingly, downsizing the study group by using the fixed ratio did not dilute the effect on QoL, as could have been expected, but rather the contrary occurred. Probably the content of the programme had a distinct effect on patients with fixed obstruction and a tangible burden of respiratory symptoms, who likely are in greatest need of integrated disease management.

The further applicability of IDM programmes such as this needs further cost-benefit studies. We tried solely to demonstrate an optimally achievable result in primary care COPD patients. Developing the study protocol and designing the treatment plans were time-consuming elements. However, since all health care workers wrote their own part of the protocol (supervised by the study coordinator), deploying the treatment plans was less of a burden, and quickly became an integrated part of daily work. Nevertheless, overall costs of the program have likely been a fraction of formal rehabilitation costs, since all patients continued to live in their own habitat, trained twice a week under supervision and once at home, while contacts with the respiratory nurse were usually every 3-6 months, depending on disease severity. Team meetings were kept at a monthly onehour session with intermittent one-to-one interdisciplinary consultations if needed (usually ten minutes once a week).

Furthermore, all health care workers reported that, contrary to common belief, it became a pleasure to work with these COPD patients, since they improved so clearly and quickly. In addition, the patients' personal goals were central to regaining a sense of control after many years of gradually losing it. Disease education and exacerbation management were excellent tools to understand the process and to remain in control for the future. Finally, the newly-found social contacts and consequent peer pressure to take control through an active lifestyle, probably are essential to make a lasting change.

Many studies have demonstrated the beneficial effects of formal COPD rehabilitation programmes in severe to very severe patients, but have also shown the difficulty in maintaining initial results in the longer term. Self-management in severe COPD patients reduced hospitalisations in Canada by almost 40\%, ${ }^{9}$ but in milder patients hospitalisations are considerably less common, and depend strongly on health care systems. By providing simpler rehabilitation programmes for less severe patients in primary care, people learn how to manage COPD in their own habitat, and health care providers are trained to coach this process directly. The negative spiral of dyspnoea and deconditioning has earlier been recognised as a central mechanism for COPD development; ${ }^{17}$ we now propose that integrated disease management can counteract this mechanism. It is likely that costs will be lower while patients are helped at an earlier stage, possibly reducing decline and disease progression in the long term. We recommend that future studies address these issues in a larger primary care population, taking into account feasibility in different health care organisations.

\section{Conclusion}

In this study, integrated disease management (IDM) improves one-year quality of life in primary care COPD patients, compared to usual care. The improvement in SGRQ was both clinically relevant and statistically significant, and was greatest in patients with an $\mathrm{FEV}_{1} / \mathrm{FVC}$ ratio $<0.7$ and an $\mathrm{MRC}$ Dyspnoea score $>2$.

\section{Conflicts of interest}

All authors declare that they have no conflict of interest with regard to this study. 
NH Chavannes et al.

\section{Acknowledgements}

The authors would like to thank all participating patients, health centers and coworkers for their efforts, which have enabled the successful completion of this study.

\section{Funding}

PICASSO for COPD, an initiative of Boehringer Ingelheim, Pfizer and Caphri Research Institute, Maastricht University.

\section{References}

1. Celli BR, MacNee W, Agusti A, et al. Standards for the diagnosis and treatment of patients with COPD: a summary of the ATS/ERS position paper. Eur Respir J 2004;23(6):932-46. http://dx.doi.org/10.1183/09031936.04.00014304

2. Casaburi R, Kukafka D, Cooper CB, Witek TJ Jr, Kesten S. Improvement in exercise tolerance with the combination of tiotropium and pulmonary rehabilitation in patients with COPD. Chest 2005;127(3):809-17. http://dx.doi.org/10.1378/chest.127.3.809

3. Calverley P, Pauwels R, Vestbo J, et al. TRial of Inhaled STeroids ANd long-acting beta2 agonists study group. Combined salmeterol and fluticasone in the treatment of chronic obstructive pulmonary disease: a randomised controlled trial. Lancet 2003;361(9356):449-56. http://dx.doi.org/10.1016/S01406736(03)12459-2

4. Calverley PM, Anderson JA, Celli B, et al. TORCH investigators. Salmeterol and fluticasone propionate and survival in chronic obstructive pulmonary disease. $N$ Engl J Med 2007;356(8):775-89. http://dx.doi.org/10.1056/NEJMoa063070

5. Herland K, Akselsen JP, Skjønsberg $\mathrm{OH}$, Bjermer L. How representative are clinical study patients with asthma or COPD for a larger "real life" population of patients with obstructive lung disease? Respir Med 2005;99(1):11-9. http://dx.doi.org/10.1016/j.rmed.2004.03.026

6. Garcia-Aymerich J, Lange P, Benet M, Schnohr P, Antó JM. Regular physical activity reduces hospital admission and mortality in chronic obstructive pulmonary disease: a population based cohort study. Thorax 2006;61(9):772-8. http://dx.doi.org/10.1136/thx.2006.060145

7. Garcia-Aymerich J, Lange P, Benet M, Schnohr P, Antó JM. Regular physical activity modifies smoking-related lung function decline and reduces risk of chronic obstructive pulmonary disease: a population-based cohort study. Am J Respir Crit Care Med 2007;175(5):458-63. http://dx.doi.org/10.1164/rccm.200607-8960C

8. Anthonisen NR, Skeans MA, Wise RA, Manfreda J, Kanner RE, Connett JE. Lung Health Study Research Group. The effects of a smoking cessation intervention on 14.5-year mortality: a randomized clinical trial. Ann Intern Med 2005;142(4):233-9.

9. Bourbeau J, Julien M, Maltais F, et al. Chronic Obstructive Pulmonary Disease axis of the Respiratory Network Fonds de la Recherche en Santé du Québec. Reduction of hospital utilization in patients with chronic obstructive pulmonary disease: a disease-specific self-management intervention. Arch Intern Med 2003;163(5):585-91. http://dx.doi.org/10.1001/archinte.163.5.585

10. http://www.ersnet.org/COPD

11. Tiep BL. Disease management of COPD with pulmonary rehabilitation. Chest 1997;112(6):1630-56. http://dx.doi.org/10.1378/chest.112.6.1630

12. Jones PW, Quirk FH, Baveystock CM, Littlejohns P. A self-complete measure of health status for chronic airflow limitation. The St. George's Respiratory Questionnaire. Am Rev Respir Dis 1992;145(6):1321-7.

13. Jones PW. St. George's Respiratory Questionnaire: MCID. COPD 2005;2(1):75-9. http://dx.doi.org/10.1081/COPD-200050513

14. Van Der Molen T, Willemse BW, Schokker S, Ten Hacken NH, Postma DS, Juniper EF. Development, validity and responsiveness of the Clinical COPD Questionnaire. Health Qual Life Outcomes 2003;1:13. http://dx.doi.org/10.1186/1477-7525-1-13

15. Kocks JW, Tuinenga MG, Uil SM, van den Berg JW, Stahl E, van der Molen T. Health status measurement in COPD: the minimal clinically important difference of the clinical COPD questionnaire. Respir Res 2006;7:62. http://dx.doi.org/10.1186/1465-9921-7-62

16. Bestall JC, Paul EA, Garrod R, Garnham R, Jones PW, Wedzicha JA. Usefulness of the Medical Research Council (MRC) dyspnoea scale as a measure of disability in patients with chronic obstructive pulmonary disease. Thorax 1999;54(7):581-6.

17. Chavannes NH, Vollenberg JJH, Schayck van CP, Wouters EFM. Effects of physical activity in mild to moderate COPD: a systematic review. $\mathrm{Br} J \mathrm{Gen}$ Pract 2002;52:574-8.

Available online at http://www.thepcrj.org 